\title{
EXPERIENCING A PROBABILISTIC APPROACH TO CLARIFY AND DISCLOSE UNCERTAINTIES WHEN SETTING OCCUPATIONAL EXPOSURE LIMITS
}

\section{DAVID VERNEZ ${ }^{1}$, SANDRINE FRAIZE-FRONTIER ${ }^{2}$, RAYMOND VINCENT ${ }^{3}$, STÉPHANE BINET $^{3}$, and CHRISTOPHE ROUSSELLE ${ }^{2}$}

\author{
${ }^{1}$ University of Lausanne and Geneva, Lausanne, Switzerland \\ Institute of Work and Health (IST) \\ ${ }^{2}$ French Agency for Food, Environmental and Occupational Health and Safety (ANSES), Maisons-Alfort, France \\ ${ }^{3}$ National Institute for Research and Security for the Prevention of Occupational Accidents and Diseases (INRS), \\ Vandoeuvre-lès-Nancy, France
}

\begin{abstract}
Objectives: Assessment factors (AFs) are commonly used for deriving reference concentrations for chemicals. These factors take into account variabilities as well as uncertainties in the dataset, such as inter-species and intra-species variabilities or exposure duration extrapolation or extrapolation from the lowest-observed-adverse-effect level (LOAEL) to the noobserved-adverse-effect level (NOAEL). In a deterministic approach, the value of an AF is the result of a debate among experts and, often a conservative value is used as a default choice. A probabilistic framework to better take into account uncertainties and/or variability when setting occupational exposure limits (OELs) is presented and discussed in this paper. Material and Methods: Each AF is considered as a random variable with a probabilistic distribution. A short literature was conducted before setting default distributions ranges and shapes for each AF commonly used. A random sampling, using Monte Carlo techniques, is then used for propagating the identified uncertainties and computing the final OEL distribution. Results: Starting from the broad default distributions obtained, experts narrow it to its most likely range, according to the scientific knowledge available for a specific chemical. Introducing distribution rather than single deterministic values allows disclosing and clarifying variability and/or uncertainties inherent to the OEL construction process. Conclusions: This probabilistic approach yields quantitative insight into both the possible range and the relative likelihood of values for model outputs. It thereby provides a better support in decision-making and improves transparency. Int J Occup Med Environ Health 2018;31(4):475-489
\end{abstract}

Key words:

Risk management, Chemical toxicity, Assessment factors, Uncertainty distributions, Probabilistic methods, Occupational exposure limits

\section{INTRODUCTION}

Since 2005 the French Agency for Food, Environmental and Occupational Health and Safety (ANSES) has been responsible for organizing an independent, multidisciplinary, collective scientific expertise for setting occupa- tional exposure limits (OELs). A dedicated independent expert committee (OEL Committee) has been set up to support the Agency in this mission.

The French system for establishing regulatory OELs is organized following 3 phases:

Received: February 1, 2017. Accepted: August 28, 2017.

Corresponding author: David Vernez, University of Lausanne and Geneva, Institute of Work and Health (IST), Rue de la Corniche 2, 1066 Epalinges, Switzerland (e-mail: david.vernez@hospvd.ch). 
- independent scientific expertise by the OEL Committee leading to recommendations of occupational exposure limits for chemical agents; this is the only phase entrusted to the Agency;

- publication by the French Ministry of Labour of a draft regulation document which includes proposals for new limit values, which may be binding or indicative;

- stakeholders consultation on these proposals and discussion among the French Steering Committee on Working Conditions (COCT). The aim of this phase is to discuss the effectiveness of the limit values and, if necessary, to determine a possible implementation timetable, according to its technical and economic feasibility.

Occupational exposure limits, as proposed by the OEL Committee, are concentration levels of pollutants in the workplace atmospheres that should not be exceeded over a determined reference period and below which the risk of impaired health is considered negligible [1]. These concentration levels are established considering a population of workers, thus excluding both the children and the elderly. Occupational exposure limits are determined based on information available from epidemiological, clinical and experimental studies. The establishment of OELs as for other reference values such as toxicity reference values (TRVs) or indoor air quality guidelines (IAQGs) usually relies on a deterministic approach [2]. This approach produces a single point estimate of the OEL by dividing the point of departure (POD), which may be the no-observed-adverse-effect level (NOAEL), the lowestobserved-adverse-effect level (LOAEL) or a benchmark dose (BMD) by the product of assessment factors (AFs). Assessment factors applied to the POD are expected to offset variability and uncertainties related to the data taken into account and their interpretation in the adjustment process. Assessment factors may reflect uncertainties related to the lack of knowledge (e.g., uncertainty related to the extent to which an effect observed in the case of animals may be transposed to humans) as well as intra or inter-individual variability. AFs may also be applied to take into account differences in exposure conditions between laboratory studies and actual worker exposure or uncertainties around the POD (LOAEL vs. NOAEL, for example). These factors may thus be broken down into inter-species, intra-species, time-related and data-related uncertainty assessment factors. The values of these factors are often sources of controversy and debate and so it is necessary to ensure that their choice is justified, transparent and not unduly influenced by risk management considerations.

It is generally assumed that AFs are values that fall within intervals of 1-10 for inter-species variability and 1-5 for variability in the population of healthy adults. The choice of an appropriate value for the AF is usually discussed by experts, based on the available data for the substance considered. Several limitations of the deterministic approach must be pointed out:

- A point estimate of a value does not adequately reflect variability and uncertainty around this value. If there is no specific or conclusive data, the experts will usually choose a prudent default value, thus often considering a "worst-case" situation.

- The multiplication of prudent assessment factors may result in an overestimated overall factor and therefore unrealistic reference values.

In order to better take into account uncertainties and/or variability in the process of OELs elaboration, it was decided to develop an ad hoc probabilistic approach [3]. The probabilistic approach intends to describe more clearly variability and/or uncertainties in yielding quantitative insight into both the possible range and the relative likelihood of values for model outputs.

A probabilistic approach, using Monte Carlo (MC) simulation, to establish OELs is investigated in this study. Its possible uses and limitations are discussed. This approach only covers substances for which a threshold effect may be assumed. Substances without a threshold (e.g., genotoxic carcinogens) have not been considered in this study. 


\section{MATERIAL AND METHODS}

\section{Uncertainties and ranges of existing assessment factors}

The determination of likely ranges and probability distribution for each assessment factor requires a high level of scientific expertise. The information that is needed cannot be obtained directly from reference material. Expert judgment is therefore used but should be supported by assumptions, publications and/or other data sources. Furthermore, when different distributions are possible for a given AF, the sensitivity analysis should be conducted in order to weight the different options [4]. A review of the literature was carried out, for each usual source of uncertainty, i.e., each AF, in the OEL context (i.e., exposure to chemical by inhalation during occupational activities), in order to define what might be their extreme values, the shape of their distributions among theoretical probability law (uniform, triangular, log-normal, etc.) and the associated parameters of these distributions (e.g., mean, standard deviation, percentiles). The available empirical data published is not always relevant to chemical substances found in occupational environments. The dataset used in this study to elaborate the AF's distributions has therefore been critically analyzed taking into account the context of the OELs' development.

\section{Inter-species assessment factor}

An inter-species assessment factor $\left(\mathrm{AF}_{\mathrm{A}}\right)$ is necessary when extrapolating from animal data to human data. Adjustment through the $\mathrm{AF}_{\mathrm{A}}$ allows for the estimation of a human equivalent dose (HED), taking into account toxicokinetic and toxicodynamic differences between species. The $\mathrm{AF}_{\mathrm{A}}$ is frequently divided into 2 subfactors: an allometric assessment factor, which takes into account differences induced by body size and related differences in metabolic rate, and species-specific differences.

In the study from Kalberlah et al. [5] on respiratory toxicants, it has been shown that when comparing LOAELs and NOAELs from 33 Agency for Toxic Substances and
Disease Registry (ATSDR) reports, for gases/liquids, in $62 \%$ of the cases, humans seemed to be more sensitive than animals. This percentage drops to $53 \%$ in the case of particles. Possible distributions for $\mathrm{AF}_{\mathrm{A}}$ were investigated through several studies in comparing animal-animal NOAEL ratios or animal-human NOAEL ratios. Vermeire et al. analyzed mouse, rat and dog data and found out that the NOAELs ratios were lognormally distributed. When adjusted for allometric scaling, their distribution had a geometric mean (GM) of about 1 and a geometric standard deviation (GSD) of 6 [6]. The author suggested that the large GSD observed was due to the variability of the NOAEL itself and that the true inter-species distribution was probably smaller. This argument was supported by results from the previous study, reporting the GSD of about 5 [7] as well as a further analysis and extension of Vermeire's data, which lead to the GSD of 4.5 [8]. Moreover, several authors proposed theoretical distributions consistent with the current worst case default factor of $10[9,10]$. More recently Schneider et al. [11] has compared toxicological data on 63 anti-neoplastic agents among 6 species. When adjusted by scaling according to the caloric demand, the inter-species ratios distributions were found to be similar and approximating 1. The GM and GSD of the resulting empirical distribution were 0.97 and 3.23 , respectively (95th percentile $=7.04$ ). A higher variability was observed by Price when analyzing anti-neoplastic data issued from the National Cancer Institute. Interspecies ratios above 10 were found in about $20 \%$ of the data points. These ratios were, however, possibly overestimated because the data was obtained from a sensitive subpopulation of cancer patients [12].

\section{Inter-individual factor}

The inter-individual assessment factor $\left(\mathrm{AF}_{\mathrm{H}}\right)$ takes into account variations in the population such as age, gender, genetics composition and nutrition. The use of specific data to assess the appropriate inter-individual variability is 
advocated whenever possible. Default values for the $\mathrm{AF}_{\mathrm{H}}$ in the general population, ranging between 3 and 10 , have still been proposed by several national and international organizations. While arbitrary, the historical value of 10 (upper default value) has subsided nowadays. The evidence to support a different value is currently lacking and it is generally considered that this value should be sufficient to protect the majority of the population against adverse health effects [5,13].

Studies have been conducted to substitute or substantiate the existing default factors. Only a few of them are based on experimental data and the ones available are mostly based on pharmaceuticals substances rather than on industrial toxicants [14]. Hattis et al. investigated the total change in the pharmacokinetics of 49 drugs in the case of healthy adults and concluded that, except for one drug, the use of a safety factor 10 would correspond to 7-9 standard deviations in populations of normal healthy adults, suggesting that the tenfold factor was too conservative [15]. The further analysis of these results showed that the inter-individual ratios, defined as the ratios of $\mathrm{P}_{50}$ and $\mathrm{P}_{5}$ plasma half-life-time distributions, were about 1.4 [6]. Considering the $5 \%$ incidence level in a given population, Hattis et al. assessed the effect of a tenfold decrease in exposure. The resulting incidences found were of $10^{-3}-10^{-4}$, depending on the substance [16].

Although several studies suggest that the factor of 10 is sufficient to take into account pharmacokinetic variability, the $\mathrm{AF}_{\mathrm{H}}$ could be underestimated. The available data was indeed obtained on healthy volunteers rather than a sensitive population and the pharmacodynamic effect is often insufficiently considered. After analyzing the available human data, Kalberlah et al. concluded that the factor of 10 may not be sufficient to cover sensitive population under all circumstances [5].

Probability distributions of $\mathrm{AF}_{\mathrm{H}}$ were also proposed by several authors, either based on the data analysis or theoretical considerations. Most of them assume a lognormal distribution. Baird et al. proposed distributions based on the data from acute toxicity and heterogeneity amongst rats. The distribution of the $\mathrm{GM}=2.7$ and the GSD $=2.3$ was obtained assuming an identical heterogeneity in the case of rats and humans, while the distribution of the $\mathrm{GM}=5.3$ and the GSD $=2.1$ was obtained assuming that humans were 1.5 times more heterogeneous than rats [7]. Swartout et al. proposed the distribution of the $\mathrm{GM}=3.16$ and the 95 th percentile of 10 , arguing that 3.16 was close to the frequently used default value of 3 and that 10 corresponded to the upper default range [10]. Slob and Pieters proposed the distribution of the $\mathrm{GM}=4$ and the 1st-99th percentile range of 2-10 [9]. This corresponds to the lognormal distribution with the median of 3 and the dispersion factor of 3 shifted by 1 unit on the right. It was assumed by the authors that the inter-species factor could not be smaller than one.

\section{LOAEL to NOAEL extrapolation factor}

Benchmark dose is considered to be the best indicator to be used as the POD to derive OELs. However, in the absence of benchmark doses, the NOAEL is generally considered to be the relevant point of departure to set an OEL. When no reliable NOAEL is available, experts have to start from the lowest-observed-adverse-effect level (LOAEL) and apply an assessment factor $\left(\mathrm{AF}_{\mathrm{L}}\right)$ to take into account the LOAEL/NOAEL ratio.

The default value of 3-10 for the $\mathrm{AF}_{\mathrm{L}}$ is frequently used, although limited data is available to support it. The LOAEL/NOAEL ratios of about 350 substances including volatile organic compounds (VOCs), alcohols and pesticides were investigated by Pieters et al. [17]. The distribution of the data suggested a lognormal distribution of the LOAEL/NOAEL ratio. The geometric means of subacute $(\mathrm{N}=95)$, subchronic $(\mathrm{N}=226)$ and chronic $(\mathrm{N}=175)$ LOAEL/NOAEL ratios found were 3.5, 4.3 and 4.5, respectively. The 95th percentiles found were of 9,16 and 11, respectively. It was shown that, although an $\mathrm{AF}_{\mathrm{L}}$ of 10 is in the order of magnitude of 
the 95th percentiles observed, the value of 10 might have been even too low.

However, these results have to be considered carefully because the LOAEL/NOAEL ratio is strongly influenced by the study design [17]. One has to keep in mind that the no-observed-adverse-effect level (NOAEL) is only a proxy of the "true" no-adverse-effect level (NAEL), which will remain unknown. This uncertainty increases with the spacing between the NOAEL and the LOAEL, and thus the dose spacing of the study. Moreover, Zarn et al. highlighted that the dose spacing (and thus the LOAEL/NOAEL ratio within a same study) generally increased with exposure duration [18].

\section{Exposure duration extrapolation factor}

The extrapolation from subchronic to chronic studies has been investigated and published for several decades. In most cases, $\mathrm{AF}_{\mathrm{S}}$ was estimated using the $\mathrm{NOAEL}_{\text {subchronic }}$ $\mathrm{NOAEL}_{\text {chronic }}$ ratio for the same species and critical effect. All authors concur with the assumption that the assessment factor is lognormally distributed, mostly because of the lognormal behavior of the NOAEL data. The extent of its distribution is, however, still a matter of debate. Reviews of published studies on subchronic to chronic assessment factor were proposed by several authors. Pieters et al. reviewed the results from early studies reporting geometric means (GM) and geometric standard deviations (GSD) of 1.4-2.3 and 2.2-3, respectively [17]. Pieter's own study, based on less homogenous data, resulted in a much wider distribution $(\mathrm{GM}=1.7$; $\mathrm{GSD}=5.6)$, suggesting that the default assessment factor of 10 could be too low.

The large variability of $\mathrm{AF}_{\mathrm{S}}$ was further highlighted by a thorough review conducted by Vermeire et al., which reported the GM and GSD ranges of 1-4.1 and 1.3-5.6, respectively (including the results already reported by Pieters) [6]. Vermeire et al. pointed out that the observed variability might have been due to the heterogeneity of the data (inter-species variation, variable subchronic exposure periods) and that the GM value could be overestimated. He suggested that the GM of 2 and the GSD of 4, corresponding to a 95th percentile of 20 , was a reasonable approximation. While it is generally agreed that the value of the predicted GM was within a narrow range, typically $1.5-2$, there were few consensuses about the possible value of the GSD. Others authors suggested GSD values notably lower than the ones proposed by Vermeire et al. The GSD of 2.5 was suggested by Baird et al., who repeated the analysis of the part of the NOAEL available data [7]. Slob and Pieters argued that the AFS might have been overestimated because it was based on the NOAEL rather than on the "true" NAEL and thus contained additional uncertainty [9]. This argument was further investigated by Bokkers and Slob, who compared subchronic to chronic ratio using the NOAEL and critical effect doses [19]. The use of critical effect doses decrease significantly the distribution GSD, leading to the 95th percentile 3.3 times lower and within the 2.9-3.3 range. It was concluded that AFs based on the NOAEL data may have lead to unnecessarily conservative values. Moreover, the 95th percentiles values observed by Bokkers and Slob were all below 10 . Studies investigating the subacute to subchronic assessment factor are limited and tend to be based on smaller datasets than subchronic to chronic studies. Unsurprisingly, the variability of the observed distributions tends also to be higher. Vermeire et al. reported the default lognormal distribution with the GM of 2 and the GSD of 4 [6]. Lower values were found by Groeneveld et al., who obtained the lognormal distribution with the GM of 1.6 and the GSD of 3.3 [20]. With the 95th percentile of 11, this distribution is consistent with the default value of 10 for extrapolation from subacute to subchronic. A detailed investigation of rats and mouse NOAEL and the LOAEL data was conducted by Zarn et al. [18], who obtained distributions for subacute to subchronic ratios consistent with Groeneveld's study. The ranges of the GM and GSD values observed were of 1.1-2.1 and 2.1-3.1, respectively. 


\section{Quality of data extrapolation factor}

The assessment factor for quality of data, the $\mathrm{AF}_{\mathrm{D}}$, takes into account the amount, quality, completeness and consistency of the available data. There is little argument to support a specific range or distribution for the $\mathrm{AF}_{\mathrm{D}}$. Some authors suggested that the $\mathrm{AF}_{\mathrm{D}}$ could run up to 100 [6]. Although, it could be argued that when confidence in the data is so poor, it will unlikely be used for deriving the OEL. Evans and Baird compared the NOAEL estimates for 35 pesticides derived from censored and uncensored datasets [21]. A substantial increase in the precision of estimates was observed when the database became more complete. A wide dispersion of the estimated assessment factor, defined as the NOAEL-censored/NOAEL-uncensored ratio, was found. Database limitations, in terms of representativeness and size, however precluded a strict probabilistic interpretation of these results.

\section{Accounting for uncertainties}

\section{in the OEL production process}

Based on the literature screening and experience of the members of the French OEL committee, a probabilistic approach, making use of a classical Monte Carlo method, was developed. As shown in the equation 1, the probabilistic approach is based on the same model as the one used for setting OELs in a deterministic way. It is assumed, however, that some (if not all) of the AFs could be uncertain or variable:

$$
\mathrm{OEL}=\frac{\mathrm{POD}}{\prod_{\mathrm{i}=1}^{\mathrm{k}} \mathrm{AF}_{\mathrm{i}}}
$$

where:

POD - chosen point of departure of OEL process (a NOAEL, LOAEL or a BMD),

$\mathrm{k}$ - number of assessment factors considered, $\mathrm{AF}_{\mathrm{i}}-$ ith assessment factor for $\mathrm{i}=1, \ldots, \mathrm{k}$.
The first step of the probabilistic approach is to assign the probability distribution (probability density function) to each uncertain AF. These distributions may be derived from chemical-specific data, when available. In such a case, the GM represents the central estimate of the available data base of chemicals; while the GSD (width) is associated with the number of chemicals in the database, the methods to estimate the ratios/quantities that form the quantitative estimate of the distribution as well as the specific characteristics of the chemical. In most cases, however, expert judgment is used for assessing the available toxicological data. In the first step, the maximum range of potential values relevant for each $\mathrm{AF}$ is specified. The probability distribution for the $\mathrm{AF}$ is then defined by selecting the shape (log-normal, uniform, triangular, etc.) and the associated parameters. In the second step, the AFs' probability distributions are propagated through the model [22]. As a general rule, these factors are assumed to be independent. In practice, this is done to simulate separately an $\mathrm{N}$ random sampling of each uncertain AFs from their respective distribution. The model is then run repeatedly $\mathrm{N}$ times, supplied by the $\mathrm{k}$ sets of $\mathrm{N}$ simulated samples. Finally, a N-sample of the studied OEL is provided, from which one can retrieve the required statistics (mean, mode, standard deviation, percentiles, etc.), as described in the Figure 1.

\section{Application to some case studies}

For this study, the approach was applied to 2 selected substances: ethylene glycol monoethyl ether (EGEE) and ethylene glycol monoethyl ether acetate (EGEEA), for which the OELs committee had already proposed some deterministic OELs [3]. Three AFs were considered, the inter-species $\mathrm{AF}$, the inter-individual $\mathrm{AF}$ and the exposure duration AF. For each of them, 100000 random draws were performed using the R Statistical Software (version 3.2). To compare both approaches, the AFs were either deterministically determined or a priori distributed. The deterministic OEL was then compared to the distribution of the OEL following the Monte Carlo approach. 


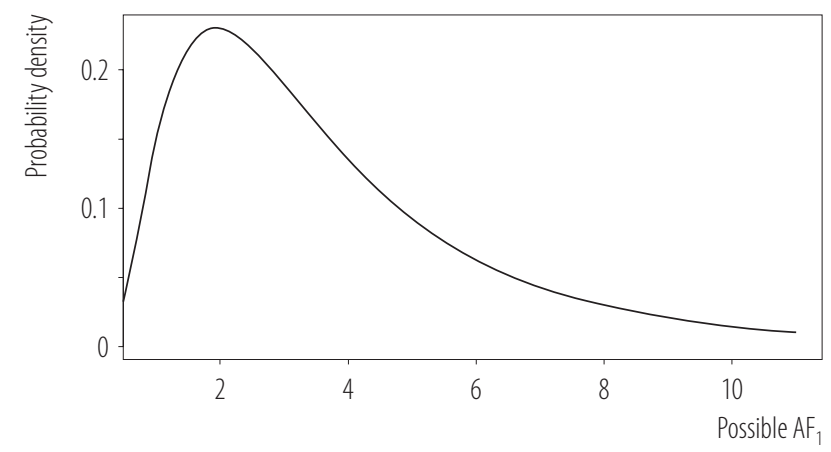

Monte Carlo simulation $\longrightarrow \quad \mathrm{af}_{11}, \quad \mathrm{af}_{12}, \quad \ldots, \quad \mathrm{af}_{1 \mathrm{~N}}$

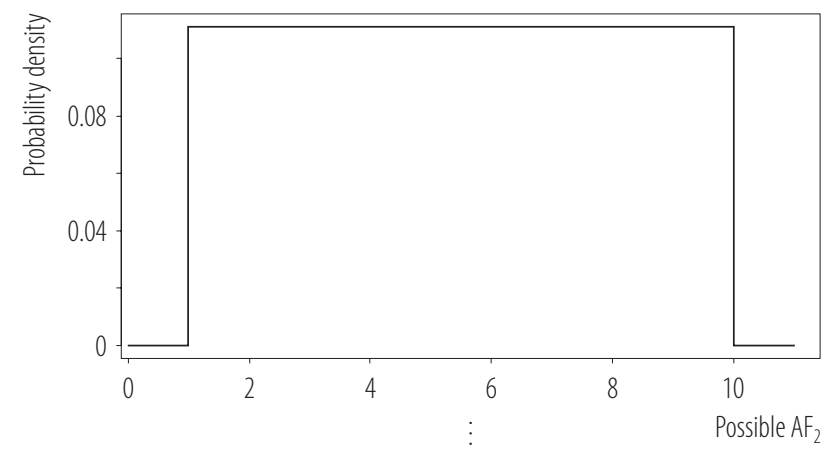

Monte Carlo simulation $\longrightarrow \quad \mathrm{af}_{21,} \quad \mathrm{af}_{22}, \quad \ldots, \quad \mathrm{af}_{2 \mathrm{~N}}$

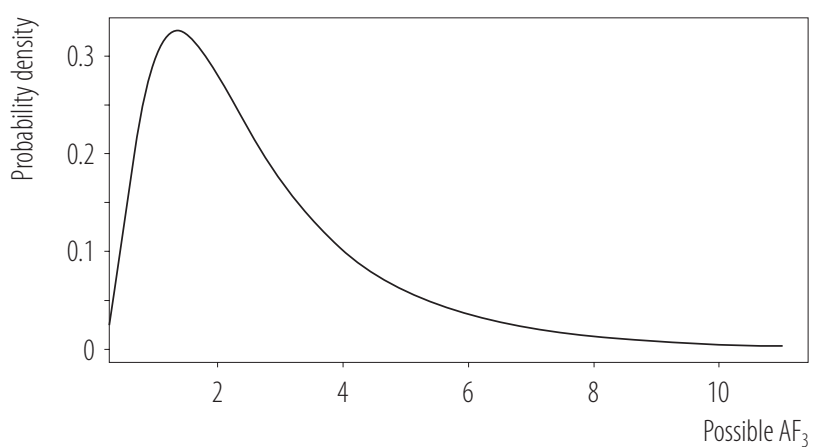

Monte Carlo simulatio
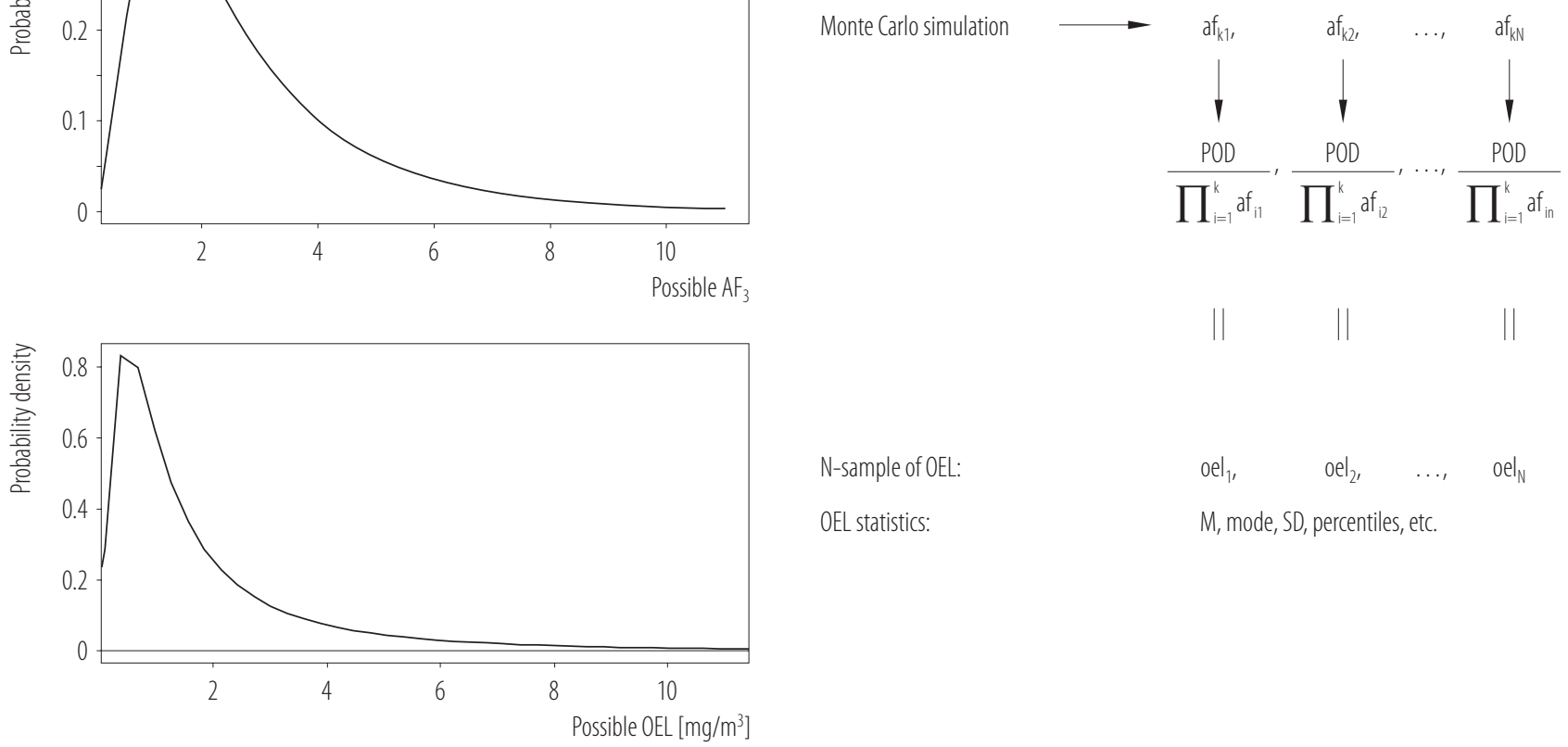

N-sample of OEL:

OEL statistics: oel ${ }_{1}$

oel $_{2}$

$M$, mode, SD, percentiles, etc.

$\mathrm{AF}$ - assessment factor; $\mathrm{af}_{\mathrm{k}}$ - assessment factor value for uncertainty $\mathrm{k}$; POD - point of departure; $\mathrm{i}$ - ith value Monte Carlo simulation run; $\mathrm{M}$ - mean; SD - standard deviation.

Fig. 1. Propagation of numerical uncertainty using Monte Carlo methods through the process of occupational exposure limits (OELs) elaboration 


\section{RESULTS}

\section{Distributions used to derive OEL}

Considering the large uncertainties reported by the literature due to the lack of knowledge about the real variability of most AFs, a pragmatic approach was chosen by the French OEL Committee [3]. An overview of its general framework is presented in the Figure 2. In this approach, the French OEL committee selects a critical effect in the key study identified through a literature review. The POD, which may be either the NOAEL/LOAEL or preferably the BMD, is derived based on the available data. Similarly to the current practices, the relevant assessment factors were considered one after the other. But rather than selecting a single $\mathrm{AF}$ value, the experts will consider a range of possible values, defined by their 5th and 95th percentiles. The choice of the appropriate AFs and their range is discussed by the experts from the OEL committee. Starting from a broad initial (default) distribution, the experts have to narrow it to its most likely range according to the current scientific knowledge about the studied substance.

The distributions obtained for individual assessment factors are combined to get the resulting distribution of the $\mathrm{AF}\left(\mathrm{AF}_{\mathrm{R}}\right)$ and subsequently, the most likely distribution of the human equivalent dose (HED). The default distributions considered by the French OEL Committee [3] are presented in the Table 1 . These distributions are derived from the assessment factor's ranges currently used by the Committee, assuming that the upper and lower values represent the most likely range of the AF. For all AFs, the upper value is set as the 95 th percentile. The lower value is set as the 5 th percentile or minimum, depending on the context.

According to the short literature analysis conducted, a lognormal or shifted lognormal distribution is assumed for all AFs except for the $\mathrm{AF}_{\mathrm{D}}$. A shifted lognormal distribution is chosen for the $\mathrm{AF}_{\mathrm{L}}$, to take into account that the LOAEL is not expected to be lower than its associated NOAEL, assuming the monotonicity in the dose-

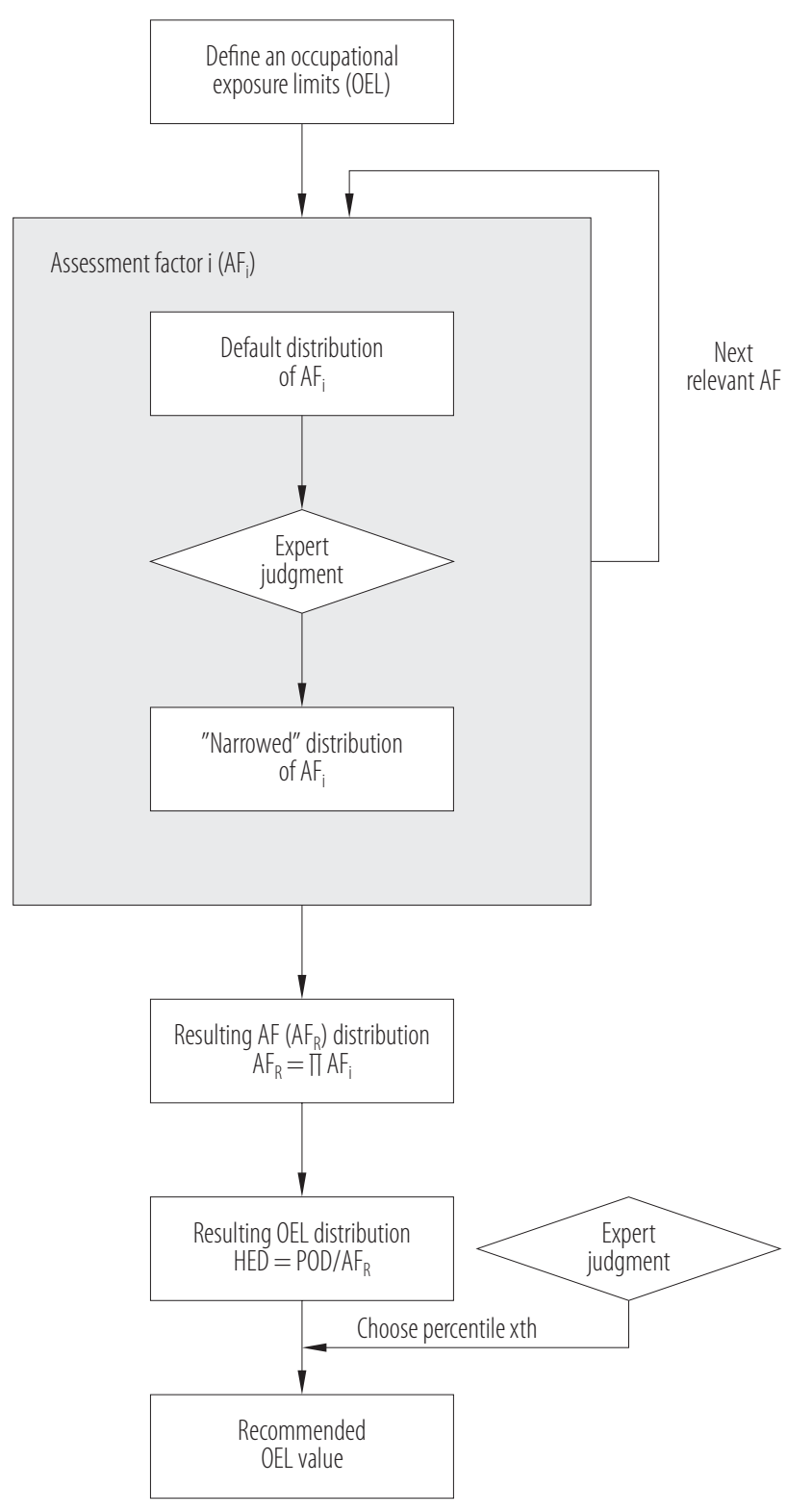

HED - human equivalent dose; POD - point of departure.

Fig. 2. Overview of the framework proposed to establish an occupational exposure limit (OEL) value based on the probabilistic approach

response relationship. A linear distribution (uniform) between 1 and 10 is chosen for the quality of data because no value is considered as more probable than the others within the uncertainty range. The default distribution for the $\mathrm{AF}_{\mathrm{A}}$ takes into account both toxicokinetic and toxico- 
Table 1. Default distributions considered by the French OEL Committee when setting assessment factors (AF) without prior information [3]

\begin{tabular}{llc}
\hline \multicolumn{1}{c}{ Assessment factor } & \multicolumn{2}{c}{ Probability distribution } \\
\cline { 2 - 3 } & \multicolumn{1}{c}{ shape } & \multicolumn{1}{c}{ parameters } \\
\hline For inter-species differences $\left(\mathrm{AF}_{\mathrm{A}}\right)$ (pharmacokinetics, pharmacodynamics) & lognormal & $\mathrm{P}_{0.05}=1 ; \mathrm{P}_{0.95}=10$ \\
For inter-individual variability $\left(\mathrm{AF}_{\mathrm{H}}\right)$ & lognormal & $\mathrm{P}_{0.05}=1 ; \mathrm{P}_{0.95}=5$ \\
For LOAEL to NOAEL $\left(\mathrm{AF}_{\mathrm{L}}\right)$ & lognormal-like* & min. $=1 ; \mathrm{P}_{0.95}=10$ \\
For differences in exposure duration $\left(\mathrm{AF}_{\mathrm{S}}\right)$ & lognormal & $\mathrm{P}_{0.05}=1 ; \mathrm{P}_{0.95}=10$ \\
For quality of data $\left(\mathrm{AF}_{\mathrm{D}}\right)$ & uniform & min. $=1 ;$ max $=10$ \\
\hline
\end{tabular}

LOAEL - lowest-observed-adverse-effect level; NOAEL - no-observed-adverse-effect level.

$\mathrm{P}$ - percentiles; min. - minimal value; $\max$ - maximal value; $\mathrm{Y}$ - transformed distribution (shifted on the right); $\mathrm{X}$ - initial distribution.

${ }^{*} \mathrm{Y} \approx \operatorname{lognormal}-$ like (min. $\left.=1 ; \mathrm{P}_{0.95}=10\right)$ if $\mathrm{Y}=1+\mathrm{X}$ with $\mathrm{X} \approx \operatorname{lognormal}\left(\mathrm{P}_{0.05}=1 ; \mathrm{P}_{0.95}=9\right)$.

dynamic differences and includes the allometric adjustment. When the allometric and/or dosimetric adjustments are already considered, for instance, when using data issued from physiologically based pharmacokinetic (PBPK) modeling, the 95th percentile of the distribution could be reduced accordingly.

Similarly to the scheme currently in use in most OELs' Committees, its probabilistic nature allows for conveying information about uncertainties up to the final recommendation. Moreover, it allows for a clear distinction between adjustments intended to cope with uncertainties and safety margins (the choice of a given percentile of the final distribution).

\section{Application to some case studies}

To establish the French OELs for the EGEE and EGEEA, the ANSES OELs committee started with the POD of $375 \mathrm{mg} / \mathrm{m}^{3}$ and $550 \mathrm{mg} / \mathrm{m}^{3}$, respectively. These values corresponded to the NOAELs for hematotoxic effects, from experimental study on rats and rabbits published by Barbee et al. [23]. The rats were repeatedly exposed by the inhalation route $6 \mathrm{~h}$ /day, 5 days/week during 13 weeks. Following its usual regular deterministic approach [1], the committee considered 3 assessment factors: inter-species differences extrapolation $\left(\mathrm{AF}_{\mathrm{A}}\right)$, inter-individual variabil- ity $\left(\mathrm{AF}_{\mathrm{H}}\right)$ and differences in exposure duration extrapolation $\left(\mathrm{AF}_{\mathrm{S}}\right)$. The $\mathrm{AF}_{\mathrm{A}}$ of 3 was considered to be sufficiently prudent for the extrapolation of hematotoxic effects. The $\mathrm{AF}_{\mathrm{H}}$ was set to 10 , which was above the usual default value in the occupational population, but was deemed necessary because of the high inter-individual variability of enzymatic metabolism (e.g., according to ethnic origin). The value of the $\mathrm{AF}_{\mathrm{S}}$ was set to 3 , to take into account the subchronic to chronic extrapolation. These choices led to the following deterministic OELs: $3.75 \mathrm{mg} / \mathrm{m}^{3}$ for the EGEE and $5.5 \mathrm{mg} / \mathrm{m}^{3}$ for the EGEEA.

Following the same reasoning through a probabilistic approach would have led to 3 lognormal distributions for the $\mathrm{AF}_{\mathrm{A}}, \mathrm{AF}_{\mathrm{H}}$ and $\mathrm{AF}_{\mathrm{S}}$, with percentiles ranges of $1\left(\mathrm{P}_{0.05}\right)-3\left(\mathrm{P}_{0.95}\right), 1\left(\mathrm{P}_{0.05}\right)-10\left(\mathrm{P}_{0.95}\right)$, and $1\left(\mathrm{P}_{0.05}\right)-3\left(\mathrm{P}_{0.95}\right)$, respectively. The resulting distributions of the equivalent HED for the EGEE and EGEEA are shown in the Figure 3 and Table 2. The values range $0.1 \mathrm{mg} / \mathrm{m}^{3}-$ $1851 \mathrm{mg} / \mathrm{m}^{3}$ and $0.1-2716 \mathrm{mg} / \mathrm{m}^{3}$, respectively. For the EGEE, the deterministic OELs of $3.75 \mathrm{mg} / \mathrm{m}^{3}$ were proposed by the OELs committee. We can observe that this value corresponds to the 9th percentile of the EGEE HED distribution. The most likely value of $5.5 \mathrm{mg} / \mathrm{m}^{3}$ (the mode) of the distribution, was about 1.5 times higher than the chosen OEL. For the EGEEA, the determinis- 

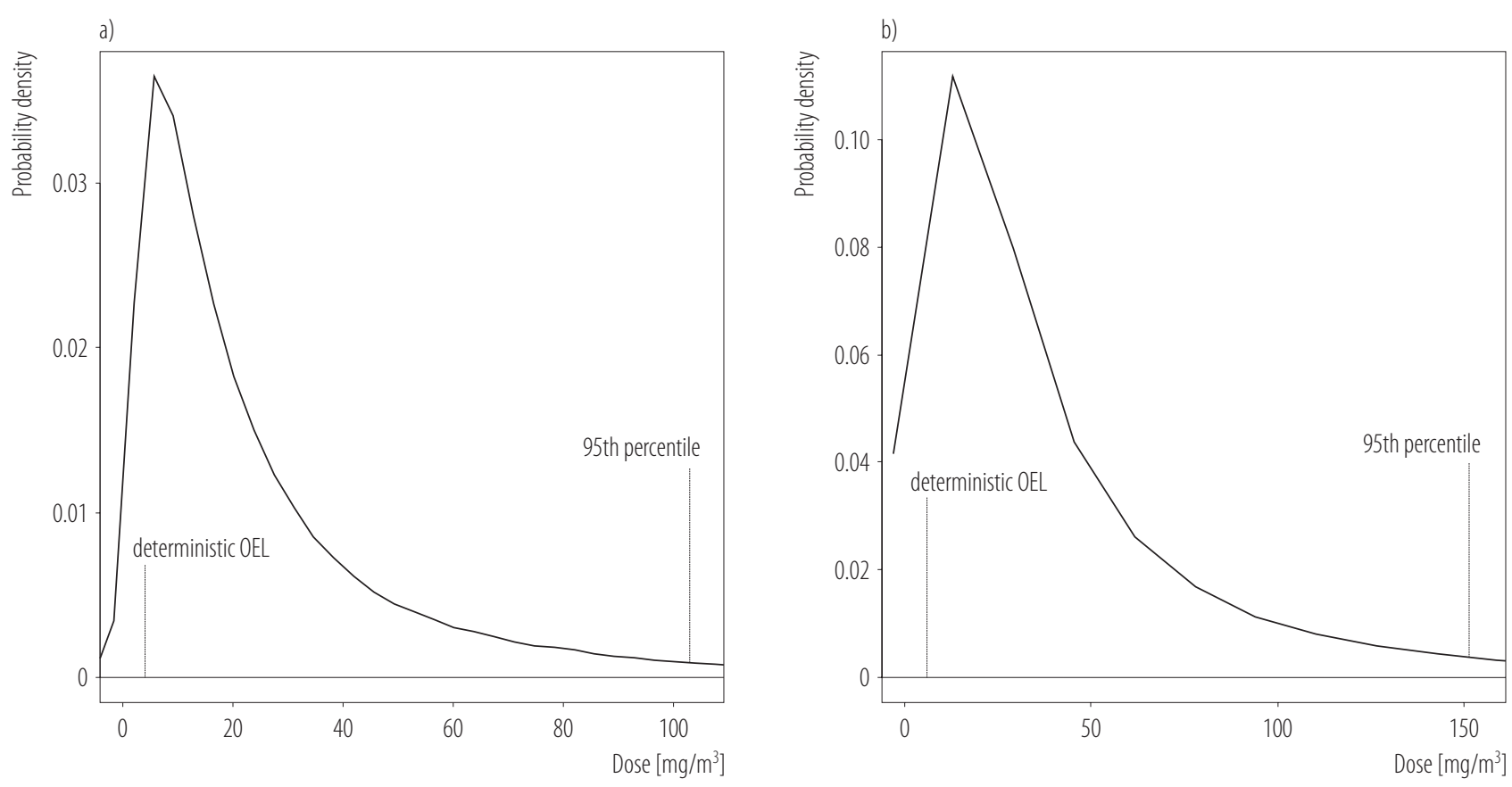

OEL - occupational exposure limit.

Fig. 3. Human equivalent dose for a) ethylene glycol monoethyl ether (EGEE), b) ethylene glycol monoethyl ether acetate (EGEEA)

Table 2. Statistics of the resulting human equivalent dose distributions for ethylene glycol monoethyl ether (EGEE) and ethylene glycol monoethyl ether acetate (EGEEA)

\begin{tabular}{lcccccccc}
\hline \multirow{2}{*}{ Substance } & \multicolumn{8}{c}{$\begin{array}{c}\text { Concentration } \\
{\left[\mathrm{mg} / \mathrm{m}^{3}\right]}\end{array}$} \\
\cline { 2 - 9 } & min. & $\mathrm{P}_{0.05}$ & $\mathrm{P}_{0.5}$ & $\mathrm{M}$ & mode $^{*}$ & $\mathrm{P}_{0.95}$ & $\max$ & SD \\
\hline EGEE & 0.1 & 2.7 & 16.8 & 30.9 & 5.5 & 103.3 & 1851.5 & 48.6 \\
EGEEA & 0.1 & 4.0 & 24.6 & 45.3 & 8.1 & 151.3 & 2715.5 & 71.3 \\
\hline
\end{tabular}

$\mathrm{P}$ - percentiles; min. - minimal value; max - maximal value; $\mathrm{M}$ - mean; $\mathrm{SD}$ - standard deviation.

* The most likely value.

tic value of $5.5 \mathrm{mg} / \mathrm{m}^{3}$ was established by the committee. According to the probabilistic approach, the most likely value of the distribution was of $8.1 \mathrm{mg} / \mathrm{m}^{3}$, corresponding to the 9th percentile of the EGEEA HED distribution.

\section{DISCUSSION}

The probabilistic approach to establish OELs is presented in this paper. In this approach, each AF used is considered to be a random variable with a probabilistic distribution based on available data and expert judgment. All the distributions of the relevant AFs are combined, using statistical techniques, to quantify the uncertainty in the final result. Several shapes of the AF's distribution are possible: lognormal, uniform, triangular. Available literature on the possible distribution of inter- and intra-species effects, ratios of LOAELs to NOAELs, and differences in acute and chronic effects suggest that most AFs tend to be approximately lognormally distributed. In the probabilis- 
tic approach, each AF is replaced by a lognormal distribution based on empirical observations to identify, median, standard deviation, and percentiles. The default distributions are to be considered as a starting point, prior to the debate among experts. Default distributions are defined by the $5-95$ th percentile range or min.-max range, assuming that $90 \%$ and $100 \%$ of the values should fall within this range, respectively. Assuming that the uncertainty factors are independent and lognormal-distributed, the logarithm of their product is being distributed as the sum of normally distributed variables; the percentiles of the resulting distribution are easily computed.

When chemical specific information is available, chemical-specific adjustment factors (CSAFs) could be used for replacing default AFs, according to the scheme adopted by the World Health Organization/International Program on Chemical Safety [24,25]. In most cases, detailed CSAFs are, however, not available and full probabilistic dosesresponse assessments [26,27] are not possible. The pragmatic approach considered by the French OEL committee adds a probabilistic dimension to the expert judgment process, disclosing the uncertainties of decision making of the OELs.

The use of the probabilistic approach has several advantages:

- By assigning a probabilistic distribution to each AF, it avoids having to systematically multiply "worst-case" situations. In the deterministic approach, very prudent assessment factors are often chosen and they are multiplied together. This results in a high overall factor that leads to a low limit value [28]. Thus, due to the multiplication of assessment factors, the choice of default values may rapidly lead to OELs that are unrelated to the relevant concentrations in terms of health. While this over-cautious approach does favor worker protection and promotes a "lowest" exposure objective, it has severe drawbacks in terms of risk prioritization for both authorities and companies.
- It avoids the ruling out of some chemicals or some studies from the OELs process. According to the methodology of the OEL Committee [1], a key study should not be used for the establishment of OELs if it is necessary to apply more than 3 assessment factors to the POD or if the total value of these factors exceeds 1000. A more adequate exclusion criteria could be used with a probabilistic, considering the extent of the uncertainty distribution for the resulting $\mathrm{AF}_{\mathrm{R}}[3]$.

- The probabilistic approach is also more transparent: instead of being a deterministic figure, the OEL would be derived based on the distribution of values that reflects the experts' committee knowledge of each factor and its variability or uncertainty.

The point estimate proposed in the deterministic approach is the result of an expert consensus that is not always visible in the final outcome. The choice of a single value of the AF, sometimes arbitrary, does not reflect the richness of the panel discussion and real uncertainty around the decision. The AFs are often mixtures of multiple concepts. They may reflect variability, and/or uncertainty adjustments. Depending on the context and available level of information, the AF value may be the most probable estimate, a prudent estimate or a median value between these 2 bounds.

Furthermore, it is not clear whether the choice of such a value reflects real variability or uncertainty related to the lack of adequate data. Disclosing the uncertainty and variability inherent to the OEL construction process should better support the decision making and, ultimately the acceptance of the final OEL value. The expert judgement and debate will still be encouraged but instead of being focused on the choice of assessment factors to be applied and the value assigned to each of these factors, it will focus on the shape of distributions and the extreme values, i.e., the lower and upper bounds of the distributions. Currently, when deriving occupational exposure values, the fraction of the population which should be protected 
is rarely specified. The incidence, reflecting the likelihood of an event occurring, is often specified for genotoxic carcinogens (e.g., $10^{-5}$ for workers), but not for other types of effects. When the BMD approach is used, the typical coverage used is $90 \%$, but this coverage relates only to the benchmark dose level (BMDL), not to the OEL value. Moreover, the degree of uncertainty and variability in the available scientific information should be explicitly considered. In the approaches described in our manuscript, the degree of uncertainty in estimating the OEL and its coverage (the probability that it provides the intended level of protection) is quantified. Therefore, we think that this approach may be useful for risk managers. It provides basis for the weighing of hazards and their uncertainty against other factors relevant for risk management decision-making.

Furthermore, moving from the deterministic approach to a probabilistic one also appears adequate in the context of the derived-no-effect level (DNEL) elaborated under the REACH regulation [29]. More and more DNELs are being developed in order to cover the requirements of REACH for new substances. One can easily anticipate that the number of chemicals to be addressed to within the REACH regulatory framework is well beyond the capability of the OELs' experts committees to produce reference values. The DNELS and OELs construction logic is often opposed as if unrelated. Providing a distribution rather than a single deterministic value would highlight the continuum existing between the 2 values. In principle, both OELs and DNEL should be issued from the same uncertainly distribution. While uncertainties are expected to be much higher in DNELS (i.e., less data available), a more conservative percentile of the distribution should be chosen, leading to a lower end value.

Some limits in using the probabilistic approach also have to be pointed out:

- The calculations described in the probabilistic approach are by nature more complex than those in the deterministic approach, but with the appropriate software and standardized probabilistic inputs, the increase in time and effort is minimal in most cases.

- Not all expert choices are considered in the probabilistic approach (e.g., selection of mode of action $(\mathrm{MoA}) /$ critical effect, critical study, quality, data gap analysis), which only reflects the uncertainty and variability of the assessment factors. Because of this, the OEL construction process should continue to put emphasis on the qualitative decision made by the experts.

The probabilistic approach is well-suited for PODs with a benchmark dose. The BMD, being probabilistic by nature, takes into account the whole range of tested doses to identify the effect instead of the deterministic choice of the NOAEL/LOAEL. The BMD and BMDL concepts are other appropriate tools to better describe the uncertainty around the point of departure (POD). Although the use of BMD is more and more frequent, it had not been included in this project which has primarily been focused on substances without enough dose-response data to derive the BMD/BMDL. The combination of probabilistic approaches for both POD and AFs is planned in the future.

How the probabilistic approach could be used by risk managers is still a matter of debate. The most likely distribution of the HED value conveys important information about its associated uncertainties, according to the current level of knowledge. Moreover, the sensitivity analysis could be encouraged so that the parameters that most influence the result are identified. As such, this information should be part of the support data available to the decision maker who will set the final regulatory value.

In France, the OELs advocated by Anses to the Ministry of Labour are discussed by stakeholders before being implemented in the regulation. The final OELS could be an indicative value or binding ones. It would be perfect 
if mandatory values were attributed to substances with a high level of confidence. With the deterministic approach, the uncertainty around the recommended OEL is not known and the overall AF is only a rough proxy of the level of confidence. When possible, management decisions should preferably be based on both lower and upper bound of the risk estimates. In the probabilistic approach, the distribution of OELS, or even the 95th percentile range, provides information on the confidence around the central value. This could be a sound basis for discussion amongst risk managers and risk assessors. These probabilistic values could also be compared to probabilistic exposure data, which is more and more frequently available in order to express risk in terms of likely impacts (value-relevant impacts) on workers.

\section{CONCLUSIONS}

In conclusion, the probabilistic approach may use all available information about quantifiable variability and uncertainty and estimate their combined influence on OELs values. It provides a fuller characterization of impact of uncertainty and variability in the range of observation and in value-relevant parameters, and therefore provides valuable advancement in hazard characterization and more informed risk management. More importantly, we hope that this paper will stimulate a gradual, but long-needed, refinement in regulatory approaches for non-cancer risk assessment.

\section{ACKNOWLEDGMENTS}

Authors wish to thank the French Agency for Food, Environmental and Occupational Health and Safety (ANSES) for its support, the members of the Expert Committee for recommending occupational exposure limits for chemical agents (OEL Committee) for their advice, as well as Billy Amzal, Patrick Breton, Marie-Laure Cointot, Mounia El Yamani and Renaud Persoons for their major contribution to the development of the methodology described in this paper.

\section{REFERENCES}

1. French Agency for Food, Environmental and Occupational Health and Safety (Anses) [Internet]. Maisons-Alfort: The Agency; 2014 Jan [cited 2017 Jan 5]. Request No.: 2009-SA0339. Expert appraisal on recommending occupational exposure limits for chemical agents. Reference document for the derivation and the measurement of exposure limit values for chemical agents in the workplace (OELs). Collective expert appraisal report. Available from: https://www.anses.fr/en/system/files/VLEP2009sa0339RaEN.pdf.

2. French Agency for Food, Environmental and Occupational Health and Safety (Anses) [Internet]. Maisons-Alfort: The Agency; 2012 Jul [cited 2017 Jan 5]. [Request No.: 2011-SA0355. Health reference values. Guidelines of analysis and selection practices. Report]. Available from: https://www.anses. fr/fr/system/files/CHIM2011sa0355Ra.pdf. French.

3. French Agency for Food, Environmental and Occupational Health and Safety (Anses) [Internet]. Maisons-Alfort: The Agency; 2014 Jan [cited 2017 Jan 5]. [Request No.: 2013-SA0235. Occupational exposure limit values. Reference document on a probabilistic approach to establishing threshold OELs. Collective expert appraisal report]. Available from: https://www.anses.fr/fr/system/files/VLEP2013sa0235Ra.pdf. French.

4. Hammonds JS, Hoffman FO, Bartell SM. An introductory guide to uncertainty analysis in environmental and health risk assessment. Report No.: ES/ER/TM-35/RI. [Internet]. Oak Ridge: SENES Oak Ridge, Inc.; 1994 Dec [cited 2017 Jan 5]. Available from: http://citeseerx.ist.psu.edu/viewdoc/ download?doi $=10.1 .1 .123 .231 \&$ rep $=$ rep1\&type $=$ pdf.

5. Kalberlah F, Schneider K, Schuhmacher-Wolz U. Uncertainty in toxicological risk assessment for non-carcinogenic health effects. Regul Toxicol Pharmacol. 2003;37(1):92-104, https:// doi.org/10.1016/S0273-2300(02)00032-6.

6. Vermeire T, Stevenson H, Peiters MN, Rennen M, Slob W, Hakkert BC. Assessment factors for human health risk assessment: A discussion paper. Crit Rev Toxicol. 1999;29(5):43990, https://doi.org/10.1080/10408449991349249. 
7. Baird SJS, Cohen JT, Graham JD, Shlyakhter AI, Evans JS. Noncancer risk assessment: A probabilistic alternative to current practice. Hum Ecol Risk Assess. 1996;2(1):79-102, https://doi.org/10.1080/10807039.1996.10387463.

8. Rennen M, Hakkert BC, Stevenson H, Bos PMJ. Data-base derived values for the interspecies extrapolation: A quantitative analysis of historical toxicity data. Comments Toxicol. 2001;7:423-36.

9. Slob W, Pieters MN. A probabilistic approach for deriving acceptable human intake limits and human health risks from toxicological studies: General framework. Risk Anal. 1998;18(6):787-98, https://doi.org/10.1111/j.1539-6924.1998. tb01121.x.

10. Swartout JC, Price PS, Dourson ML, Carlson-Lynch HL, Keenan RE. A probabilistic framework for the reference dose (probabilistic RfD). Risk Anal. 1998;18(3):271-82, https:// doi.org/10.1111/j.1539-6924.1998.tb01294.x.

11. Schneider K, Oltmanns J, Hassauer M. Allometric principles for interspecies extrapolation in toxicological risk assessment - Empirical investigations. Regul Toxicol Pharmacol. 2004; 39(3):334-47, https://doi.org/10.1016/j.yrtph.2004.03.001.

12. Price PS, Keenan RE, Swartout JC. Characterizing interspecies uncertainty using data from studies of antineoplastic agents in animals and humans. Toxicol Appl Pharmacol. 2008;233(1):64-70, https://doi.org/10.1016/j.ta ap.2008.03.026.

13. Vermeire T, Pieters MN, Rennen M, Bos PMJ. Probabilistic assessment factors for human health risk assessment: A practical guide. Report No.: 601516 005. Bilthoven: RIVM, TNO; 2001.

14. Hattis D, Baird S, Goble R. A straw man proposal for a quantitative definition of the RfD. Drug Chem Toxicol. 2002;25(4):403-36, https://doi.org/10.1081/dct-120014793.

15. Hattis D, Erdreich L, Ballew M. Human variability in susceptibility to toxic chemicals - A preliminary analysis of pharmacokinetic data from normal volunteers. Risk Anal. 1987;7(4):415-26, https://doi.org/10.1111/j.1539-6924.1987. tb00479.x.
16. Hattis D, Banati P, Goble R. Distributions of individual susceptibility among humans for toxic effects. How much protection does the traditional tenfold factor provide for what fraction of which kinds of chemicals and effects? Ann N Y Acad Sci. 1999;895:286-316, https://doi.org/10.1111/ j.1749-6632.1999.tb08092.x.

17. Pieters MN, Kramer HJ, Slob W. Evaluation of the uncertainty factor for subchronic-to-chronic extrapolation: Statistical analysis of toxicity data. Regul Toxicol Pharmacol. 1998;27(2):108-11, https://doi.org/10.1006/rtph.1997.1196.

18. Zarn JA, Engeli BE, Schlatter JR. Study parameters influencing NOAEL and LOAEL in toxicity feeding studies for pesticides: Exposure duration versus dose decrement, dose spacing, group size and chemical class. Regul Toxicol Pharmacol. 2011;61(2):243-50, https://doi.org/10.1016/j.yrt ph.2011.08.004.

19. Bokkers BG, Slob W. A comparison of ratio distributions based on the NOAEL and the benchmark approach for subchronic-to-chronic extrapolation. Toxicol Sci. 2005; 85(2):1033-40, https://doi.org/10.1093/toxsci/kfi144.

20. Groeneveld CN, Hakkert BC, Bos PMJ, Heer C. Extrapolation for exposure duration in oral toxicity: A quantitative analysis of historical toxicity data. Hum Ecol Risk Assess. 2004;10(4):709-16, https://doi.org/10.1080/ 10807030490484318.

21. Evans JS, Baird S. Accounting for missing data in noncancer risk assessment. Hum Ecol Risk Assess. 1998;4(2):291-317, https://doi.org/10.1080/10807039891284352.

22. Hoffman FO, Gardner RH. Evaluation of uncertainties in radiological assessment models. In: Till JE, Meyer HR, editors. Radiological assessment. A textbook on environmental dose analysis. Report No.: NUREG/CR-3332. ORNL-5968. Washington, D.C.: NRC Office of Nuclear Reactor Regulation; 1983. p. 11-1-55.

23. Barbee SJ, Terrill JB, DeSousa DJ, Conaway CC. Subchronic inhalation toxicology of ethylene glycol monoethyl ether in the rat and rabbit. Environ Health Perspect. 1984;57:157-63, https://doi.org/10.1289/ehp.8457157. 
24. Dankovic DA, Naumann BD, Maier A, Dourson ML, Levy LS. The scientific basis of uncertainty factors used in setting occupational exposure limits. J Occup Environ Hyg. 2015;12 Suppl 1:S55-68, https://doi.org/10.1080/15459624.2015.1060325.

25. World Health Organization [Internet]. Geneva: The Organization, 2014 [cited 2017 Jan 5]. Guidance document on evaluating and expressing uncertainty in hazard characterization. Available from: http://www.who.int/ipcs/methods/ harmonization/areas/hazard_assessment/en.

26. Chiu WA, Slob W. A unified probabilistic framework for dose-response assessment of human health effects. Environ Health Perspect. 2015;123(12):1241-54, https://doi. org/10.1289/ehp.1409385.

27. Van der Voet H, Slob W. Integration of probabilistic exposure assessment and probabilistic hazard characterization.
Risk Anal. 2007;27(2):351-71, https://doi.org/10.1111/j.15396924.2007.00887.x.

28. Naumann BD, Weideman PA. Scientific basis for uncertainty factors used to establish occupational exposure limits for pharmaceutical active ingredients. Hum Ecol Risk Assess. 1995;1(5):590-613, https://doi.org/10.1080/1080703950 9380049.

29. European Chemicals Agency [Internet]. Helsinki: The Agency, 2012 [cited 2017 Jan 5]. Report No.: ECHA-2010G-19-EN. Guidance on information requirements and chemical safety assessment. Chapter R.8: Characterisation of dose [concentration]-response for human health. Available from: https://echa.europa.eu/documents/10162/13632/ information_requirements_r8_en.pdf.

This work is available in Open Access model and licensed under a Creative Commons Attribution-NonCommercial 3.0 Poland License - http://creativecommons.org/ licenses/by-nc/3.0/pl/deed.en. 